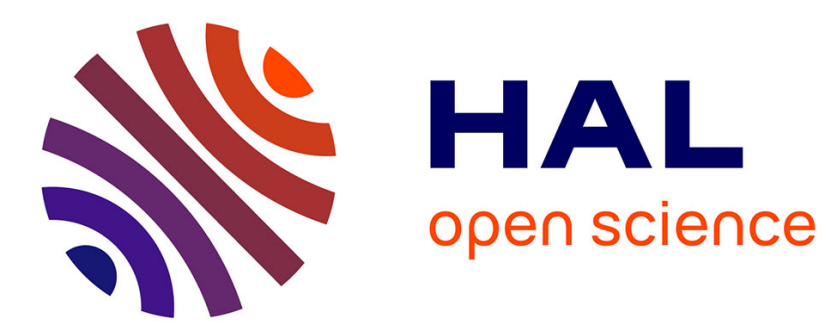

\title{
Dynamiques identitaires à l'échelle du temps : une étude de cas chez les enseignants d'Éducation Physique et Sportive
}

Thérèse Roux-Perez

\section{- To cite this version:}

Thérèse Roux-Perez. Dynamiques identitaires à l'échelle du temps: une étude de cas chez les enseignants d'Éducation Physique et Sportive. Science \& motricité: Revue scientifique de l'Association des Chercheurs en Activités Physiques et Sportives, 2005, 56, pp.75 - 96. 10.3917/sm.056.0075. hal-01716390

\author{
HAL Id: hal-01716390 \\ https://hal.science/hal-01716390
}

Submitted on 23 Feb 2018

HAL is a multi-disciplinary open access archive for the deposit and dissemination of scientific research documents, whether they are published or not. The documents may come from teaching and research institutions in France or abroad, or from public or private research centers.
L'archive ouverte pluridisciplinaire HAL, est destinée au dépôt et à la diffusion de documents scientifiques de niveau recherche, publiés ou non, émanant des établissements d'enseignement et de recherche français ou étrangers, des laboratoires publics ou privés. 


\section{DYNAMIQUES IDENTITAIRES À L'ÉCHELLE DU TEMPS : UNE ÉTUDE DE CAS CHEZ LES ENSEIGNANTS D'ÉDUCATION PHYSIQUE ET SPORTIVE}

Thérèse Roux-Perez

De Boeck Supérieur | «Movement \& Sport Sciences »

2005/3 n 56 | pages 75 à 96

ISSN 1378-1863

ISBN 2804147681

Article disponible en ligne à l'adresse :

https://www.cairn.info/revue-science-et-motricite1-2005-3-page-75.htm

\section{Pour citer cet article :}

Thérèse Roux-Perez, « Dynamiques identitaires à l'échelle du temps : une étude de cas chez les enseignants d'Éducation Physique et Sportive », Movement \& Sport Sciences 2005/3 ( $\mathrm{n}^{0}$ 56), p. 75-96.

DOI $10.3917 / \mathrm{sm} .056 .0075$

Distribution électronique Cairn.info pour De Boeck Supérieur.

(C) De Boeck Supérieur. Tous droits réservés pour tous pays.

La reproduction ou représentation de cet article, notamment par photocopie, n'est autorisée que dans les limites des conditions générales d'utilisation du site ou, le cas échéant, des conditions générales de la licence souscrite par votre établissement. Toute autre reproduction ou représentation, en tout ou partie, sous quelque forme et de quelque manière que ce soit, est interdite sauf accord préalable et écrit de l'éditeur, en dehors des cas prévus par la législation en vigueur en France. Il est précisé que son stockage dans une base de données est également interdit. 


\section{Dynamiques identitaires à l'échelle du temps : une étude de cas chez les enseignants d'Éducation Physique et Sportive}

Roux-Perez Thérèse $e^{(1)}$

\section{RÉSUMÉ}

L'article appréhende les processus en jeu dans la construction de l'identité professionnelle au travers de représentations permettant aux enseignants d'EPS de fonder et de rationaliser leurs choix professionnels. La dimension temporelle privilégiée dans cette étude souligne le caractère complexe et dynamique de l'identité professionnelle : elle met en relief des cohérences provisoires, laissant place à d'éventuels ajustements, lorsque surviennent des changements institutionnels, humains ou contextuels dans le parcours des enseignants. Ces derniers aménagent et recomposent alors certains éléments constitutifs de leur identité en fonction des situations, pour s'adapter et construire un monde cohérent entre contraintes, ressources et valeurs. L'orientation des actions et les nouvelles formes d'engagement sont justifiées par des représentations professionnelles en évolution.

Au niveau méthodologique, deux entretiens espacés de six ans ont été proposés à cinq sujets. Les résultats présentés portent sur une étude de cas. Ils montrent dans quelle mesure les interactions entre le sujet et les contextes traversés au fil du temps, objet de transactions multiples, conduisent à des formes de recompositions identitaires qui confirment le caractère à la fois stable et mouvant des identités professionnelles.

Mots-clés: identité professionnelle, représentations, dynamiques, expériences singulières, temps.

(1) IUFM des Pays de la Loire, Nantes 


\title{
Processes of change in professional identity over time: physical education (PE) teachers, a case study
}

\begin{abstract}
This article examines the processes involved in the construction of the professional identity of teachers of physical education. It looks at the attitudes and perceptions of professional roles which enable P.E. teachers to establish and rationalize their professional choices.

Special focus is given to the function of time in emphasizing the complex and changing nature of professional identity. It is seen that time can give a temporary cohesion to identity which P.E. teachers may then adjust whenever institutional, human or contextual changes occur in their careers.

When such changes take place, teachers modify and reconstruct certain aspects of their identity to adapt and build a coherent environment which is defined by professional constraints, individual resources and personal values. Changing professional attitudes and conceptions lead teachers to adjust their initiatives and adopt new patterns of involvement.

Five subjects were interviewed at a six-year interval. The results focus on one case study and show how over time, different aspects of interaction between the individual and his professional context can determine changes in identity. This would confirm both the stable and changing nature of professional identity.
\end{abstract}

Key-words : professional identity, attitudes and perceptions of professional roles, processes of change, individual experience, time factors.

\section{Introduction}

L'approche présentée dans cet article s'inscrit dans une réflexion sur la construction de l'identité professionnelle des enseignants d'EPS, envisagée comme un processus complexe et dynamique qui fait interagir l'évolution de la personne, l'évolution du métier d'enseignant et celle de la discipline. En effet, durant les vingt dernières années, la volonté institutionnelle de placer l'élève au centre des préoccupations éducatives a induit la transformation des pratiques professionnelles : construction de projets pédagogiques, traitement didactique des Activités Physiques Sportives et Artistiques (APSA), réflexion sur les contenus à enseigner et sur les modalités d'évaluation, mise en place de procédures pédagogiques nouvelles (différenciation, évaluation formative, pédagogie du contrat, etc.). Cette évolution a été vécue de façon très différente par 
les enseignants d'EPS : nombre d'entre eux se sont inscrits dans la dynamique alors que d'autres, se sentant en décalage avec les compétences professionnelles nouvelles exigées par l'institution, ont eu tendance à résister à ce changement en estimant que l'EPS était en train de " perdre son identité ». Ces différences d'interprétation ont engendré des positions relativement contrastées au sein du groupe professionnel. L'étude s'attache à comprendre ce phénomène à l'échelle individuelle.

Dans un premier temps, l'article aborde la construction de l'identité professionnelle : a) au travers de représentations permettant de fonder et de rationaliser les choix professionnels ; $b$ ) en tant que processus complexe, intégrant ajustements et réorientations ; c) dans une perspective diachronique mettant en relief l'importance des dynamiques identitaires. La méthodologie décrite prend en compte cette orientation résolument qualitative. Enfin, les résultats présentés portent sur une étude de cas. Ils montrent dans quelle mesure les interactions entre le sujet et les contextes traversés au fil du temps, objet de transactions multiples, conduisent à des formes de recompositions identitaires confirmant le caractère à la fois stable et mouvant des identités professionnelles.

\section{Des représentations constitutives de l'identité professionnelle}

Les enseignants d'EPS véhiculent un ensemble de valeurs et de croyances, partagées avec certains autres enseignants, compatibles avec leurs pratiques professionnelles ou personnelles et repérables à travers leurs représentations du métier, des autres et d'eux-mêmes (Couturier et Duret, 2000 ; Roux-Perez, 2001). En construisant ces représentations, ils réduisent d'éventuelles dissonances avec leur environnement humain (les autres acteurs) et institutionnel (les textes officiels). Les théories de la consistance cognitive (Festinger, 1957 ; Heider, 1958 ; Kiesler, 1971) en lien avec celles des représentations sociales (Abric, 1976 ; Jodelet, 1984 ; Moscovici, 1961) éclairent cette approche. En effet, envisagées comme des formes de connaissances porteuses de valeurs, les représentations sociales donnent sens à la pratique et légitiment une certaine « vision du monde ».

Par ailleurs, les représentations sont structurées autour d'un noyau central (Abric, 1976 ; Flament, 1987) auquel sont associés des éléments périphériques. Le noyau central, très résistant au changement, offre un caractère de cohérence et de stabilité à la représentation ; dans l'étude, il est perceptible au niveau des permanences qui traversent l'ensemble du discours sur la durée de l'enquête. Le système périphérique, plus souple 
et plus sensible aux effets de contexte, permet l'intégration dans la représentation des variations individuelles liées à l'histoire personnelle et déterminées par des expériences spécifiques ; ce système est mis en relief à travers la modification d'énoncés, en lien avec la situation nouvelle dans laquelle se trouve le sujet.

Ainsi, les représentations professionnelles, considérées comme l'ensemble des idées que partagent les individus sur un métier donné, sont d'abord fonctionnelles (Blin, 1997). Elles permettent aux acteurs de comprendre et d'agir sur la réalité selon un principe d'économie cognitive favorable à la résolution de problèmes quotidiens au moindre coût. Elles définissent les identités professionnelles et protègent la spécificité des groupes intra et inter professionnels. Elles orientent les conduites et guident les pratiques professionnelles par attente, anticipation, sélection, filtrage des informations par rapport à la réalité pour rendre conforme les pratiques à la représentation. Enfin, elles permettent, a posteriori, de justifier les prises de position et les pratiques professionnelles. Par ailleurs, les représentations professionnelles sont spécifiques : elles sont construites par des acteurs dans les actions et interactions professionnelles qui les contextualisent. En ce sens, elles prennent appui sur des « objets » ${ }^{(2)}$ significatifs dans le champ professionnel considéré.

Il est donc intéressant de repérer l'émergence d'un certain nombre de représentations professionnelles permettant aux enseignants de fonder, de justifier et de rationaliser leurs prises de positions vis-à-vis du métier et de la logique institutionnelle pour tenter de comprendre comment ils construisent leur monde professionnel et lui donnent sens. De ce point de vue, les représentations peuvent être considérées comme des matrices d'action constitutives des identités professionnelles (Barbier, 1996 ; Camilleri, 1990 ; Dubar, 1991 ; Barbier et Galatanu, 1998 ; Tap, 1980).

\section{L'identité professionnelle : un processus complexe et dynamique}

Dans cette approche, l'identité personnelle est envisagée comme l'ensemble des représentations et des sentiments qu'une personne développe à propos d'elle-même. C'est aussi « ce qui permet de rester le même,

(2) L'analyse des textes officiels de 1967 à 2000 permet de repérer certains objets professionnels tels que le projet pédagogique, les contenus d'enseignement, l'évaluation, le statut de l'élève, l'apprentissage, les modalités de groupement, etc. Ils constituent des « indicateurs » significatifs pour rendre compte des représentations professionnelles des enseignants interrogés. 
de se réaliser soi-même et de devenir soi-même, dans une société et une culture donnée, et en relation avec les autres. » (Tap, 1998, p. 65). En tant que processus complexe et dynamique, elle se constitue à l'articulation de trois axes principaux plus ou moins en tension. Tout d'abord, l'individu préserve le sentiment de rester le même au fil du temps et doit nécessairement s'adapter, en fonction de changements plus ou moins souhaités et/ou contrôlés : l'itinéraire professionnel intègre cet axe continuité/ changement à travers un couplage entre histoire du sujet et changements du contexte professionnel. Par ailleurs, l'individu élabore une image de soi en relation (accord, tension, contradiction) avec celles que, selon lui, les autres lui attribuent : cela conduit à un sentiment de reconnaissance ou de non reconnaissance d'autrui essentiel dans la construction identitaire. Enfin, l'individu fait en sorte de conserver une cohérence interne (unité) tout en développant une relative diversité à travers de multiples facettes sur lesquelles il peut s'appuyer pour s'adapter à des situations changeantes.

L'identité professionnelle prend appui sur le « socle » de l'identité personnelle et y intègre des composantes professionnelles : pour trouver un équilibre, l'individu se représente la discipline, le métier, les pratiques professionnelles et s'engage dans l'action. Or, s'impliquer revient à mettre en jeu une part importante de soi. L'individu s'engage en fonction des valeurs, des idéaux portés par son système de représentations et s'implique durablement dans le secteur qu'il s'est choisi, si cet engagement lui donne un sentiment de contrôle de la situation et une forme de reconnaissance sociale (Mias, 1998). L'implication au travail est donc révélatrice des rapports que le sujet établit avec son environnement professionnel. De ce point de vue, prendre en considération les modes d'implication privilégiés par les enseignants d'EPS à l'intérieur ou à l'extérieur du système scolaire, c'est tenter de comprendre ce qui fait sens pour eux, donne une cohérence à leur action et fonde, au moins en partie, leur identité.

En fait, l'identité, formée au sein d'un réseau d'interactions, est envisagée comme un processus qui intègre les différentes expériences de l'individu tout au long de la vie. Elle présente un aspect multidimensionnel, acceptable pour le sujet s'il conserve par ailleurs, la conscience de son unité et de sa continuité. Enfin, chacun dispose d'une "marge de manœuvre » pour faire face aux clivages intérieurs et aux contradictions institutionnelles. Les stratégies mises en œuvre à cette fin permettent d'entrer dans une dynamique en développant de nouveaux registres de pensée et d'action. 


\section{Des dynamiques identitaires à l'épreuve du temps}

Dans l'étude, l'identité est envisagée dans une dimension temporelle (1994-2000) au cours de laquelle l'enseignant construit des cohérences provisoires, laissant place à d'éventuelles adaptations (Dubar, 2000 ; Lahire, 1998 ; Lautier, 2001, Peyronie, 1998 ; Roux-Perez, 2001). En effet, la question des dynamiques identitaires s'inscrit dans une perspective diachronique ; elle intègre les diverses expériences du sujet et la signification qu'il accorde aux transformations de son environnement social et professionnel (Barbier, 1996). En fonction des événements, les acteurs aménagent leur définition de soi en relation à autrui ; ils opèrent des ajustements pour faire face aux variations des situations et aux enjeux qu'elles suscitent, entre nouvelles aspirations et ressources singulières.

Si la modification de repères dans l'environnement professionnel (contexte institutionnel et humain) donne lieu à des formes de réappropriation, les changements peuvent aussi être liés directement à l'histoire du sujet qui ne lit plus alors l'environnement de la même manière et modifie en partie ses représentations pour redonner sens à son action. Ainsi, les dynamiques identitaires font l'objet de compromis permettant de gérer une perspective historique (permanence de la personne) et des changements institutionnels, humains, contextuels. L'enseignant agit par rapport aux opportunités qu'il découvre et auxquelles il ajuste ses comportements (Crozier et Friedberg, 1977). Les résultats de son action l'amènent parfois à reconsidérer sa position, à repenser ses choix. Il aménage et recompose les éléments constitutifs de son identité en fonction des situations et des interactions, pour s'adapter et construire un monde cohérent entre contraintes, ressources et valeurs. L'orientation des actions et les nouvelles formes d'engagement sont alors justifiées par des représentations professionnelles qui peuvent se transformer au fil du temps.

La méthodologie utilisée dans l'enquête éclaire cette évolution des identités professionnelles au travers de discours recueillis à six ans d'intervalle chez le même sujet.

\section{Matériel et méthodes}

\section{Étude préalable}

Une première enquête (1994) a porté sur un échantillon de quinze enseignants d'EPS du second degré d'un même département, formés sur la 
base des textes de $1967^{(3)}$ et choisis selon des critères délibérément contrastés en fonction du sexe, du statut, de l'établissement d'affectation, de la stabilité dans le poste et des formes d'engagement (dans l'établissement, en milieu fédéral, dans la formation). Cette première approche du groupe professionnel s'est réalisée à partir d'entretiens semi-directifs. Une analyse de contenu a permis : a) de repérer des éléments significatifs dans les énoncés des enseignants ; b) de faire émerger le noyau central des représentations à partir des permanences qui traversent l'ensemble du discours ; c) de caractériser différents profils d'enseignants en fonction des modes d'implication privilégiés au sein ou à l'extérieur du système scolaire et des représentations professionnelles dominantes. Dans un deuxième temps des "cartes de représentations » ont été construites pour chaque groupe à partir des différents " indicateurs » professionnels ; elles intègrent les explications, les rationalisations qui permettent à chaque sujet de donner sens à son "monde " professionnel. Ces « cartes » dévoilent un réseau de significations révélateur de la manière dont l'individu construit une cohérence dans le but de rester équilibré. Les représentations issues des discours des enseignants interrogés portent sur : a) le parcours professionnel et le système de valeurs sous-jacent ; b) les prises de position sur les «indicateurs » fournis par l'analyse des textes officiels ; c) les éléments de rationalisation utilisés par les enseignants pour conserver un équilibre cognitif ; ils sont organisés autour de l'évolution de l'EPS et des représentations de l'élève (Roux-Perez, 2003).

\section{Recueil des données}

Cinq des enseignants interviewés lors de l'enquête de 1994, très représentatifs des profils identitaires construits à l'issue de ce travail ${ }^{(4)}$, ont été ré-interrogés en 2000. Cette approche a permis, par un suivi des enseignants sur plusieurs années, de mieux appréhender la dynamique identitaire à travers les permanences, les infléchissements, les modifications, voire les ruptures.

(3) Instructions officielles du ministre aux professeurs et maîtres d'éducation physique et sportive, Ministère de la Jeunesse et des Sports, Circulaire du 19 octobre 1967 (Éducation Nationale - Jeunesse et sports).

(4) Ces différents profils sont présentés dans un article intitulé «Identité professionnelle et modes d'implication privilégiés chez les enseignants d'EPS » (2003) Revue Les Sciences de l'Éducation. Pour l'Ère Nouvelle, vol 36, 4, 37-68. 
Les cinq enseignants choisis ont reçu la retranscription intégrale du premier entretien (1994) avant la passation du deuxième ; celui-ci a été structuré par un guide comportant une première série de questions liée aux trois axes constitutifs de l'identité : continuité et changement, soi et autrui, unité (cohérence) et diversité. La première question : "qu'est-ce qui vous semble avoir changé sur le plan professionnel depuis vos dernières réponses, il y a six ans ? " permettait au sujet de se situer dans sa trajectoire (articulation continuité/changement) en faisant le point sur les éventuels changements personnels (état de santé par exemple) ou liés au contexte professionnel (mutation, modifications au sein de l'établissement ou de l'équipe EPS, etc.) et sur les perceptions, sentiments qui l'accompagnaient. Les deux questions suivantes invitaient à la définition de soi pour soi et de soi pour autrui : "pourriez-vous vous définir en tant que prof d'EPS ? " et "cela vous suffit-il pour vous définir en tant que personne? "Elles permettaient de repérer les articulation soi/autrui, unité/diversité qui fondent l'identité. Ces questions étaient suivies de la proposition suivante: "professionnellement, avez-vous le sentiment d'être reconnu ou pas par les autres? ». Cette large référence à autrui renvoyait aussi bien au contexte de la classe (élèves) qu'à celui de l'établissement, des différents acteurs du système scolaire (collègues, parents, personnel de direction) ou hors système scolaire (clubs, associations, municipalité).

Une deuxième série de questions portait sur la représentation de la discipline pour le sujet (et sur la représentation de soi à l'intérieur de cette discipline). Tout d'abord une approche de l'identité disciplinaire était proposée à travers une demande très ouverte : " pourriez-vous donner trois mots qui pour vous caractérisent l'EPS actuellement? ». Puis le sujet était invité à expliciter ses réponses. Suivait une invitation à décrire " $c e$ que serait un élève physiquement éduqué », permettant l'émergence de représentations sur le rôle de la discipline, sur celui de ses enseignants vis-à-vis des acquisitions de l'élève et sur le type de savoirs privilégiés, etc. Enfin, la question: "vous sentez-vous en phase ou décalé par rapport à l'évolution de la discipline ? » conduisait à repérer l'importance accordée aux textes officiels, le degré de dissonance avec la conception « officielle » de la discipline et les éléments de justification ou de rationalisation qui l'accompagnaient.

Une troisième série de questions concernait les représentations portant sur le métier. Deux questions à choix multiple ordonné étaient proposées aux sujets durant de déroulement de l'entretien. La première portait sur «l'idéal professionnel » abordé à travers le choix de trois items dans un référentiel de compétences issu des travaux de Paquay et 
Wagner (1996) ; la deuxième invitait l'enseignant à choisir trois activités professionnelles dont il se sentait le plus proche dans l'exercice de sa profession ${ }^{(5)}$. L'explicitation des réponses permettait de repérer le sens que les enseignants accordaient aux formules choisies et les réseaux de représentations qui s'y rattachaient. La question: "qu'est-ce qui est important, pour vous, dans ce métier ? » portait sur les valeurs du métier et sur la motivation de l'enseignant pour l'accomplir.

Enfin, la pratique professionnelle était abordée par une proposition prenant en compte le contexte de travail à un niveau très concret : "si vous faisiez une lettre à un remplaçant, que lui diriez-vous pour l'aider au niveau de sa pratique dans cet établissement? ". Cette entrée avait pour but d'identifier les priorités de l'enseignant: centré sur ses classes, sur sa discipline, ouvert à l'équipe et s'appuyant sur le projet EPS, ouvert à l'établissement, etc. Enfin, les deux dernières questions abordaient une dimension plus large de l'individu en intégrant ce qui contribuait à son équilibre en dehors du travail : "quels sont les domaines qui vous intéressent en dehors du champ professionnel ? » Il s'agissait de recentrer l'entretien sur la personne, de mieux comprendre sa diversité et le degré de cohérence de ses différentes facettes. L'entretien se terminait sur la question de l'avenir professionnel pour mieux saisir le degré de consonance de l'individu vis-à-vis de ce métier et les éventuelles stratégies qui l'accompagnaient.

\section{Méthode d'analyse}

Les entretiens ont été traités à partir d'une analyse structurale du discours (Demazière et Dubar, 1997) en repérant : a) les séquences ou épisodes du récit qui éclairent le parcours professionnel et en soulignent la dynamique ; b) les actants ou personnages qui interviennent dans le récit et avec lesquels s'établissent des systèmes de relations ; c) les propositions ou arguments (représentations) destinés à convaincre l'interlocuteur, à défendre son point de vue (valeurs, identité pour soi et pour autrui, rapport à l'institution). L'intérêt de cette démarche consistait à ne pas se limiter aux seuls énoncés de discours mais à prendre en compte la chronologie, les relations et les effets d'argumentation s'inscrivant dans une structure singulière.

(5) Educateur, acteur-comédien, bricoleur, animateur, entraîneur, concepteur, technicien, accompagnateur-guide, militant, autre. 
L'analyse s'est centrée sur le jeu entre permanence et changement. Certaines transformations entre 1994 et 2000 ont pu être repérées au niveau : a) des modes d'implication internes ou externes au système scolaire ; b) du rapport identité pour soi/identité pour autrui ; c) des représentations et valeurs liées au métier ; d) des représentations professionnelles liées à la demande institutionnelle.

\section{Résultats}

La présentation d'une étude du cas a été choisie pour rendre compte de ces dynamiques identitaires à l'échelle du temps.

\section{Présentation de Brigitte en 1994 : une cohérence par la pratique compétitive}

\section{Eléments du parcours scolaire et professionnel}

Lors du premier entretien, en 1994, Brigitte est âgée de 38 ans. Elle situe son désir de devenir enseignante d'EPS dès l'entrée au collège pour "faire partager sa passion du sport » et ressembler à certains enseignants d'EPS qu'elle perçoit comme très différents des autres : " on pouvait discuter plus facilement, ils étaient plus ouverts et plus proches ». Au lycée, la rencontre d'une enseignante (Sylvie) qui est aussi son entraîneur dans le club de Basket-ball où elle joue, conduit à un processus d'identification et la conforte dans ses choix professionnels. En difficulté sur le plan scolaire et risquant d'être orientée dans une filière qu'elle juge dévalorisée, elle envisage, dès la fin de la classe de seconde, sur les conseils avisés de Sylvie, la préparation du Professorat-Adjoint d'EPS. Des années plus tard, elle juge sa formation initiale pertinente, car «centrée sur les APS et sur le concret du terrain ». Titulaire du diplôme, elle est nommée à 22 ans dans le lycée où elle était élève. En 1994, elle y enseigne depuis 17 ans.

\section{Sens du métier et formes d'implication privilégiées}

En 1994, certaines valeurs traversent le discours de Brigitte : sa motivation pour le métier est très liée à une valorisation de l'action : "être sur le terrain, toucher le ballon, se dépenser... ». L'image de « l'élève idéal » qui : "s'entraîne, s'investit, se donne à fond, a même envie de se faire mal pour améliorer sa note » conforte cette position. Par ailleurs, sa forte implication au sein de l'association sportive (AS) du point de vue des pratiques compétitives lui donne une place particulière dans le lycée. Les résultats des 
équipes en $\mathrm{UNSS}^{(6)}$ sont reconnus par le chef d'établissement qui apprécie la dynamique qu'ils suscitent dans le lycée et l'image qu'ils en donnent au dehors. Enfin, l'essentiel de son implication reste extérieure au système scolaire. Elle passe beaucoup de temps dans le club où elle évolue en tant que joueuse de niveau national. Parallèlement, entraîneur d'une équipe de jeunes, elle se forme en préparant des diplômes fédéraux.

Les représentations de l'évolution disciplinaire montrent un certain décalage avec sa propre conception de l'EPS : la formule " papier-crayon, bureau, calculatrice, ordinateur » est évoquée avec un certain humour pour souligner la dérive intellectuelle de la discipline ; de ce point de vue, le projet pédagogique est ressenti comme " formel et loin $d u$ concret $d u$ jeu ». De la même manière, la formation continue est valorisée si elle reste très pratique (centrée sur les APS) ou ouverte sur de nouvelles technologies (vidéo, informatique, etc.) qui peuvent relayer l'enseignant et donner à l'élève une connaissance plus précise de ses résultats.

Pour réduire la dissonance entre ce qu'elle valorise et ce que les textes préconisent, Brigitte développe différents types d'arguments qui prennent appui sur des représentations récurrentes dans le discours. Deux points semblent cristalliser les prises de position : un regard sur les enseignants d'EPS qui suivent l'évolution de la discipline, un autre sur les élèves dont elle décrit un certain rapport à l'EPS, proche du sien. Un regard critique est porté sur les enseignants qui «utilisent des termes nouveaux pour dire la même chose » ou qui n'ont pas fait leur travail au niveau du collège: "c'est au collège que l'on devrait travailler dans le sens des textes, au lycée c'est trop tard... les élèves arrivent en seconde, ils devraient être tous sur le même mode de fonctionnement, et ce n'est pas le cas... ». Enfin, "les collègues qui préparent les élèves en fonction des orientations actuelles » doivent se rendre à l'évidence : cela ne change rien car «les élèves oublient vite... donnent priorité à la note... et bloquent sur la formule papier-crayon ». Peu habitués à observer (au moyen de fiches), ils veulent avant tout jouer. En revanche, ils sont intéressés par la démonstration vidéo, que Brigitte utilise en gymnastique et en sports-collectifs, parce que c'est " un rapport complet et juste de ce qu'il faudrait faire et c'est plus fiable que les fiches ».

Ainsi, apparaît une organisation de l'EPS fondée sur le jeu, la pratique et la démonstration. Tout ce qui touche à l'évaluation formative, à la réflexion sur l'action n'a pas vraiment de sens, de même que l'objectif

(6) Union Nationale du Sport Scolaire 
annoncé dans la loi d'orientation (1989): «mettre l'élève au centre du système ». Pour conclure et réduire la dissonance, Brigitte évoque sa bonne volonté mais son manque de temps (lié à un surinvestissement à l'extérieur). Les perspectives professionnelles envisagées se dessinent à l'extérieur de l'enseignement de l'EPS. Elle ne tient pas à passer le $\mathrm{CAPEPS}^{(7)}$ interne et préfère être intégrée à l'ancienneté. Son souhait serait d'obtenir un poste au SUAPS(8), où elle fait quelques vacations, sur un profil de « spécialiste sport collectif » ou d'intégrer un poste administratif en UNSS.

\section{Un parcours entre permanence et changement}

En 2000, Brigitte travaille toujours dans le même lycée mais un événement majeur a bousculé sa vie. Suite à un accident en 1998 et à un arrêt de travail de plusieurs mois, elle doit cesser toute pratique physique. Immédiatement, sont évoqués les changements en terme $d$ 'investissement :

"c'est vrai que ça a changé dans la mesure où je me suis posé des questions, où je ne peux plus m'investir comme je m'investissais sur le plan physique ou pratique. Donc, les changements qui ont eu lieu, c'est au niveau de mon investissement personnel».

Dans la mesure où l'essentiel de l'investissement était en Association Sportive et en club, il est intéressant de regarder la manière dont Brigitte reconstruit son monde et (re)donne sens à son action.

\section{Au niveau de l'AS de l'établissement : de la spécialisation à la polyvalence}

En 1994, l'intérêt de Brigitte pour la dimension compétitive au sein de l'AS du lycée était évident. En Basket-ball, elle retrouvait des joueurs et joueuses du club et pouvait partager avec eux ce plaisir de la pratique sportive. En 2000, l'AS est toujours un élément important dans sa vie mais l'enjeu est devenu très différent : abandon du basket-ball (versant compétitif) pour développer une "AS de masse " (versant loisir) qui, semble-t-il, intéresse un plus grand nombre d'élèves. De plus, dans certaines activités, Brigitte sollicite l'aide d'intervenants extérieurs. Elle n'est donc plus forcément au centre du dispositif mais le fait vivre d'une autre manière, gérant ainsi ses problèmes de santé. Il n'est plus question

(7) Certificat d'Aptitude au Professorat d'Education Physique et Sportive

(8) Service Universitaire des Activités Physiques et Sportives 
de simplement reproduire des logiques de club en milieu scolaire mais d'ouvrir sur des activités diversifiées et, pour certaines d'entre elles, nouvelles dans l'établissement.

\section{Au niveau du club : ne plus entraîner mais accompagner les matchs}

En 1994, Brigitte avait commencé une formation fédérale en Basket-ball pour devenir entraîneur à plus haut niveau. Ce projet, en cours de réalisation, ne l'intéresse plus. En revanche, en 2000, elle garde une implication dans le club et s'occupe du suivi de l'équipe féminine lors des matchs, au moyen de fiches d'observation. Cette activité lui permet de garder le contact avec son club considéré comme une " seconde famille » et de rester proche de Sylvie, cette enseignante « idéale » qui l'a influencée dans son choix professionnel, tout en assumant une incapacité à jouer au plus haut niveau de pratique.

\section{Rapport entre identité pour soi et identité pour autrui}

Au niveau des élèves, Brigitte se sent perçue comme une "prof pas hyper stricte et qui procède par jeu ». Elle exprime aussi une certaine tolérance envers ceux qui n'auraient pas envie de travailler, à condition qu'ils ne dérangent pas le cours : "on peut pas en vouloir aux élèves qui s'investissent peu. Tout le monde peut pas être bon en éducation physique». Ces propos la situent assez loin des finalités de l'EPS. Ainsi, dans la conduite du cours, elle ne se focalise pas sur l'engagement des élèves. Si elle en faisait une priorité, cela la conduirait à varier ses formes d'interventions pédagogiques. En effet, bien que se disant « sensible aux élèves qui ont des difficultés scolaires ", elle ne semble pas rechercher, du moins dans les discours, des situations d'apprentissage adaptées aux différences interindividuelles En fait, Brigitte caractérise son type d'enseignement comme étant essentiellement basé sur le jeu :

« en sport-co, je fais peu de technique individuelle. Je procède énormément par jeu, jeux à thèmes, consignes à respecter, pour les faire progresser sur le plan collectif, sachant que de toute façon, on en a ni le temps, ni le matériel pour affiner la technique... Et je pense être dans le vrai par rapport à l'attente des élèves ».

Par ailleurs, elle se sent "plutôt bien perçue » par les collègues des autres disciplines, notamment lors des conseils de classe. Enfin, si son investissement au sein de l'établissement ne s'est pas accru, dans l'équipe EPS les choses ont changé : au-delà de son implication dans le cadre de l'AS, elle contribue activement à l'élaboration du projet pédagogique. 
Cette nouvelle inscription dans la dynamique de l'équipe modifie positivement le regard porté par ses collègues sur son activité professionnelle.

\section{Représentations et valeurs liées au métier}

Lors de l'entretien de 2000, Brigitte exprime une motivation plus élevée qu'en 1994 pour l'enseignement de l'EPS. Les raisons évoquées sont essentiellement la variété des activités physiques et la diversité des classes. La «bonne entente de l'équipe » joue aussi un rôle non négligeable.

Le métier d'enseignant d'EPS est perçu comme proche de celui d'éducateur, au sens de "spécialiste des APS qui apporte des connaissances spécifiques », de celui d'animateur «de jeu ». L'association avec le métier de guide pouvant ponctuellement "donner des conseils mais en dehors $d u$ cadre de l'EPS » est moins directe. Enfin, les représentations de "l'enseignant idéal » dénotent d'une continuité forte autour de valeurs déjà repérables en 1994 : a) il «s'adapte rapidement au contexte du cours » ; b) il "fait progresser les élèves pour qu'ils aient la meilleure note au bac »; c) il "développe d'autres relations avec les élèves grâce à la pratique sportive ».

De ce point de vue, si Brigitte devait accueillir un remplaçant, les informations porteraient sur trois points essentiels : les activités déjà pratiquées avec telle ou telle classe (et pas les contenus), le " comportement des élèves par rapport aux activités, aux autres élèves ou au professeur pour éviter des problèmes de discipline ou de relation » et enfin, il faudrait " insister sur l'évaluation par rapport à ce qu'on a fait dans le cadre du projet ». Ainsi, trois mots organisent le rapport à l'EPS : APSA, relation et évaluation. Il y a là une continuité certaine avec les modes de fonctionnement antérieurs et la persistance d'un noyau central dans la représentation sur le métier.

\section{Évolution des représentations professionnelles liées à la demande institutionnelle}

Inspectée peu de temps avant le premier entretien en 1994, Brigitte avait indiqué sa difficulté à s'adapter à une évolution disciplinaire dont elle ne voyait pas l'utilité. En 2000, elle revient sur ses positions :

" il y a cinq ou six ans, j'avais été inspectée et je m'étais complètement remise en question parce que l'inspecteur m'avait démontré que j'étais pas complètement dans le faux mais j'étais quand même pas dans le vrai, à ce moment-là par rapport à la discipline, au niveau des textes ». 
Entre 1994 et 2000, on peut repérer un changement de représentations portant sur certains indicateurs prélevés dans les textes : 1 'importance du projet pédagogique, l'intérêt accordé aux savoirs d'accompagnement de l'action $^{(9)}$, la place de l'élève dans l'apprentissage, le statut des APSA, l'évaluation formative et certificative. Trois de ces indicateurs sont explicités ci-après.

\section{Le projet pédagogique : d'un simple phénomène de mode à une réelle efficacité}

Dans l'entretien de 1994, Brigitte se sent décalée par rapport aux textes : "les textes : ils sont là et on est obligé d'en tenir compte. C'est évident. Mais je t'avouerai franchement que j'ai pas tout détaillé parce qu'au début, ça m'a paru rébarbatif ". A ce moment-là, elle associe la pression des textes à l'élaboration du projet pédagogique au sein de l'équipe EPS :

"quand il a fallu se mettre au projet et compagnie, j'ai trouvé qu'on s'éloignait un peu de ce que je pensais que ça devrait être l'enseignement de l'éducation physique au lycée, parce que ça devenait moins pratique pour les élèves au niveau de la pratique sportive et qu'ils s'y retrouvaient pas ».

De ce point de vue, elle ne dissocie pas la mise en cohérence des contenus et des modes d'évaluation (phase de construction faite par l'équipe d'enseignants) et les mises en œuvres concrètes au sein du cours face aux élèves. Conceptualiser les contenus, mener une réflexion didactique sur les APS support, c'est alors s'éloigner des pratiques et du jeu, ce qui motive son enseignement. Elle énonce une implication « minimale »: " par le biais des réunions qu'on a eu pour mettre en place le projet, j'ai quand même réussi à me mettre au goût du jour et à saisir ce qu'il fallait faire. » L'utilisation de l'expression " au goût du jour » montre le caractère éphémère qu'elle attribue aux textes, perçus comme étroitement liés à des phénomènes de mode.

En 2000, la perception a changé et l'implication est toute autre : «ce qui a changé pour moi, sur le plan déjà personnel, c'est que j'ai un investissement supérieur à celui que j'avais, au niveau de l'équipe péda, surtout cette année, en ce qui concerne la refonte du projet ». Dans ce cadre, l'apport de Brigitte concernant le traitement informatique des données est un atout supplémentaire pour réaliser l'objectif commun : "avoir une banque de

(9) Ces savoirs d'accompagnement de l'action correspondent à la troisième finalité de l'EPS : "accès aux connaissances relatives à l'organisation et à l'entretien de la vie physique » (1996). 
données dans laquelle chacun peut aller se servir pour faire ses séances. C'est ça, notre gros projet ». Ainsi, par le biais d'un outil qui l'intéresse, les contenus d'enseignement sont abordés d'une autre manière, avec la perspective d'un résultat concret au niveau des séances. De plus, à cette occasion, elle devient "personne-ressource » auprès de ses collègues et peut approfondir son attrait pour les nouvelles technologies déjà évoqué en 1994 à travers l'intérêt porté à l'outil vidéo.

\section{Évaluation formative et fiches d'observation : d'un outil encombrant à un gain de temps pour la pratique}

Dans l'entretien de 1994, Brigitte revient souvent à l'emploi des fiches $\mathrm{d}^{\prime}$ observation, qu'elle rattache directement et avec un certain scepticisme à l'évolution de l'EPS et au projet pédagogique: "les fiches, c'est bien, mais il faut prendre le temps de les gérer, il faut que ce soit clair à gérer sinon c'est pas fiable !». Cela sous-entend le travail à réaliser par l'enseignant entre deux séances pour un meilleur suivi de l'élève ou du groupe, travail qu'elle ne fait pas par "manque de temps ». De ce point de vue, l'utilisation de ces fiches ne peut pas servir pour d'éventuelles régulations dans les apprentissages et ne peut avoir de sens pour les élèves ; c'est d'ailleurs l'argument développé : « la question qui revient chez les élèves c'est : "quand est-ce qu'on joue? ». C'est pour ça que moi, je me dis : "il y a quelque chose qui ne va pas à ce niveau-là ». Ainsi, ne prenant pas en considération les enjeux de formation pour l'élève sous-jacents à ce type d'outil (appropriation de repères sur l'action, connaissance de soi, visualisation des progrès, objectifs de transformation, etc.), Brigitte préfère se donner d'autres priorités, davantage liées à ce qui fonde sa motivation à enseigner, à savoir la pratique et la relation directe aux élèves : "j'ai l'impression de perdre du temps par rapport à ce que je pourrais leur apporter en les conseillant, en les corrigeant... ». L'argument ultime devient alors le manque de temps durant le cours pour mettre en place une telle procédure : "fonctionner comme ça c'est très bien, mais il faudrait quatre heures par classe et par semaine pour que ça serve à quelque chose ».

Dans le deuxième entretien en 2000, Brigitte n'envisage plus les choses de la même manière : "on est passé de fiches d'observations faites par les élèves, qui étaient difficilement gérables, à des fiches d'observations mais plus dans l'objectif d'aider les élèves et non pas de s'en servir pour une évaluation ». On assiste donc à un changement de perspective : $c^{\prime}$ est du point de vue de l'élève que les fiches d'observation sont utilisées. Mais dans le discours qui suit apparaît un changement de statut de la fiche dont l'utilisation permet essentiellement de transmettre des informations aux élèves. 
Dans cette perspective, elles deviennent un réel atout pour l'enseignant : "ça donne un peu plus de temps pendant les cours, pour faire... de la pratique...Ils savent à l'avance ce sur quoi ils seront évalués, la façon dont ils seront évalués, ils peuvent le lire à tous les cours ». Cependant, on repère aussi un changement dans l'utilisation de certaines fiches d'autoévaluation en début de cycle qui : " permettent aux élèves de se situer au départ d'une part, et ce qui nous permet à nous, de voir si les élèves se situent par rapport à ce $q u^{\prime}$ on leur propose ». Bien que l'enjeu formatif soit perceptible à ce niveau, la pertinence de ces outils semble davantage liée à un gain de temps pour la pratique qu'à une possibilité de meilleur suivi de l'apprentissage de l'élève.

\section{L'évaluation certificative : toujours une priorité}

En 1994, la question du manque de temps est rattachée à une priorité : l'évaluation certificative. Brigitte la déplace du côté des élèves pour en prouver la pertinence, renforçant ainsi les logiques consuméristes de certains d'entre-eux vis-à-vis de l'EPS ; de plus, cette position lui permet alors de relativiser l'utilité des autres types de savoirs préconisés dans les textes comme les savoirs d'accompagnement de l'action :

"on a les élèves à préparer pour le bac, il y a des priorités... pas pour moi, mais pour l'élève, la priorité, c'est d'avoir des notes et les meilleures performances possibles. Donc moi, en terminale, je fonctionne pour les améliorer à une performance, pour qu'ils aient une note correcte et c'est ce qu'ils nous demandent, en fait! ».

Cette donnée varie très peu en 2000. Il est même question de commencer cette préparation dès la seconde : "même si pour eux, c'est abstrait et loin. Et nous, on a, à partir de la classe de seconde, l'objectif du bac... Donc, on les prépare à ça ». En effet, Brigitte voit comme un atout l'augmentation du coefficient au baccalauréat et en fait un vecteur de motivation pour tous les élèves : "c'est important pour les élèves parce que malgré tout, ça leur permet, quand ils ont un investissement logique et correct, sans être de super athlètes, ils récupèrent malgré tout des points ». Cet aspect s'avère primordial. En ce sens, elle reproche à l'un de ses remplaçants d'avoir failli à sa tâche en ne notant pas les élèves: "c'est gênant parce que, quand on reprend ses élèves ... on sent qu'il y a un malaise parce que certains s'attachent beaucoup à l'évaluation et à la note ». Ainsi, l'évaluation certificative traverse le discours, sans qu'il ne soit jamais question des contenus enseignés ou appris. 


\section{Discussion}

\section{Des dynamiques inscrites dans une expérience singulière}

Les dynamiques et recompositions identitaires à l'échelle du temps sont relativement perceptibles au niveau de cette étude de cas. En 2000, Brigitte ne pratique plus en compétition et consacre davantage de temps à son engagement en EPS. La crainte de ne plus enseigner cette discipline redonne un sens à son implication professionnelle. Certains indicateurs attestent d'une transformation dans sa perception de l'évolution de l'EPS. D'autres ont peu évolué : il s'agit des éléments qui fondent son attachement au métier et restent liés à sa propre histoire. Ce regain d'intérêt professionnel là où d'autres abandonneraient, pour raisons de santé, est renforcé par la possibilité d'entrer dans la dynamique de l'équipe EPS à travers l'outil informatique. L'utilisation de ce «chemin détourné » pour améliorer le projet pédagogique et le rendre plus opérationnel, en élaborant un corpus de situations pédagogiques " prêtes à l'emploi » (banque de données) représente une oportunité. Par ailleurs, le contexte humain de l'établissement est favorable à ce réajustement professionnel. Un consensus minimum existe au sein de l'équipe pour maintenir un certain confort d'enseignement ; l'élaboration d'un projet pédagogique intégrant l'explicitation des contenus et des modalités d'évaluation pour le baccalauréat répond à l'exigence institutionnelle. De plus, un respect des différences interindividuelles laisse à Brigitte la possibilité de modifier ses pratiques dans le cadre de l'AS, d'entrer dans la réflexion commune avec sa particularité et d'y trouver une place qui lui convient.

Enfin, cette enseignante relativement critique vis-à-vis de l'évolution disciplinaire en 1994 transforme son rapport à l'institution avec une évolution des représentations (système périphérique) : projet pédagogique, évaluation formative et troisième finalité de l'EPS prennent sens. En revanche, le noyau central des représentations évolue peu : pratique, jeu, technique des APS, évaluation certificative restent des ancrages forts, rattachés à son histoire sportive. Brigitte est donc sur le chemin du changement, à condition de garder les valeurs qui ont construit sa vie.

On assiste à des glissements / adaptations à l'intérieur d'un contexte qui s'est modifié face à une implication sportive qu'il n'est plus question d'assumer de la même manière. Pourtant, la continuité est assurée par le système de valeur, central et inscrit/construit dans l'histoire de cette 
enseignante qui reste "sportive de cœur » mais délaisse une identité d'entraîneur de club qu'elle transférait dans le cadre de l'association sportive du lycée. Malgré tout, en 2000, « l'élève physiquement éduqué » ressemble étrangement à un pratiquant sportif : il sait s'échauffer, a construit des habiletés motrices, des connaissances techniques et tactiques qui lui permettent de maîtriser l'APS. La référence de Brigitte reste liée à son histoire : elle défend les valeurs du sport, en cherchant à les adapter au mieux au contexte de l'EPS. Centrée sur les pratiques et la dimension relationnelle avec les élèves, elle rejoue inconsciemment son propre rapport aux savoirs scolaires et sportifs.

\section{Des éléments constitutifs des dynamiques identitaires}

Plus largement, au terme d'une étude longitudinale (1994-2000) portant sur cinq enseignants (dont Brigitte), on repère que l'articulation entre discipline, groupe professionnel d'appartenance et individu n'est jamais définitive et se construit dans une dimension temporelle, intégrant à la fois : a) des événements, des changements, des moments de crises et des recompositions ; b) des modes de relation à autrui (élèves, enseignants d'EPS dans l'équipe, autres enseignants, chef d'établissement) ; c) un rapport à l'institution et ses recommandations (inspecteur, textes officiels). En fait, tour à tour les acteurs adoptent tous les points de vue. Confrontés à des identités et à des relations sociales diversifiées, ils s'engagent dans des expériences les amenant à parcourir des logiques différentes, faisant l'objet d'arrangements multiples. En effet, accords et arrangements avec soi-même et avec les autres permettent de mettre à distance l'expérience passée, et de la revisiter en fonction de nouveaux critères.

On repère également que si certains éléments personnels ou contextuels se modifient, et prennent part au processus même de l'expérience professionnelle et personnelle, l'individu conserve une " permanence à soi », cohérence irréductible qu'il défend, y compris quand la situation semble tout à fait décalée. Par exemple, alors que l'EPS est une discipline visant le développement des conduites motrices de tous les élèves, Brigitte accepte que certains ne participent pas au cours car "tout le monde ne peut pas aimer le sport et la compétition ».

Par ailleurs, l'enquête nous montre l'importance de certains éléments « équilibrateurs » qui rendent le contexte favorable aux réorganisations, assurant ainsi les conditions d'un changement réussi. Tout d'abord, le «contexte établissement » : il s'agit de la place donnée à la 
discipline, de la reconnaissance accordée à ceux qui la font et des espaces potentiels à investir, déterminants pour que l'enseignant opère des réajustements et trouve du sens à son action, au sein de la collectivité éducative. Les attentes et représentations du chef d'établissement envers la discipline et ses acteurs participent largement à cette perception du contexte professionnel.

Par ailleurs, le «contexte humain de l'équipe d'EPS» apparaît comme un élément incontournable, plus ou moins favorable aux adaptations : consensus et tolérance, respect des singularités invitent Brigitte à réinvestir différemment le travail collectif après un accident qui met fin à son fort engagement sportif.

Enfin, le «contexte personnel» peut faciliter ces ajustements à travers : a) la mise en jeu combinée de traits de personnalité (capacité d'adaptation, d'autoanalyse, de distanciation) ; b) la diversité des facettes liée aux expériences multiples et conduisant à la construction de compétences potentielles (Lahire, 1998) ; c) la cohérence du Moi à travers des représentations qui permettent de réduire d'éventuelles dissonances et de préserver les valeurs qui mobilisent le sujet.

Ainsi, les cinq itinéraires choisis dans l'étude montrent le caractère dynamique de l'identité où l'acteur et le contexte se déterminent mutuellement. Dans un contexte professionnel donné, l'individu construit plus ou moins consciemment un équilibre provisoire ; il suffit qu'un élément de l'environnement ou qu'un élément inhérent au sujet se modifie pour que l'ensemble se réorganise. La perte du sentiment d'équilibre amène l'individu à se repositionner pour redonner sens à son contexte: il (ré)interprète alors ses propres conduites, qu'il explique et justifie, les rationalisant à travers des représentations professionnelles; celles-ci lui permettent d'établir un autre rapport au métier, à ses acteurs et, plus largement, à l'institution.

On retrouve donc, à travers ces analyses, ce qui fonde l'identité personnelle, synthèse provisoire d'un jeu de tensions entre continuité et changement, soi et autrui, cohérence et diversité. Les interactions entre le sujet et les contextes traversés au fil du temps, objet de transactions multiples, donnent à l'identité professionnelle son caractère complexe et dynamique. 


\section{Bibliographie}

ABRIC, J.C. (1976). Jeux, conflits et représentations sociales. Cousset : Del Val.

BARBIER, J.M., \& GALATANU, O. (1998). Action, affects et transformation de soi. Paris : PUF.

BARBIER, J.M. (1996). De l'usage de la notion d'identité en recherche. Education permanente, 128, 11-26.

BLIN, J.F. (1997). Représentations, pratiques et identité professionnelle. Paris : L'Harmattan.

CAmilleri, C., KASTERSZTEIN, J., LiPIANSKy, E.M., MAlewSKA-PeYRE, H., TABOAdALEONETTI, I \& VASQUEZ, A. (1990). Stratégies identitaires. Paris : PUF.

COUTURIER, C., \& DURET, P. (2000). Enseigner l'EPS : identité professionnelle à l'épreuve $d u$ sport. Paris : Centre National d'Études EPS et Société.

Crozier, M., \& FrIEdBerG, E. (1977). L'acteur et le système. Paris : Seuil.

DemazièRE, D., \& DubAR, C. (1997). Analyser les entretiens biographiques. Paris: Nathan.

DUBAR, C. (1991). La socialisation : construction des identités sociales et professionnelles. Paris : Armand Colin.

DUBAR, C. (2000). La crise des identités, l'interprétation d'une mutation. Paris : PUF.

FESTINGER, L. (1957). A theory of cognitive dissonance. Evanston Ill : Row \& Peterson.

FLAMENT, C. (1987) Pratique et représentations sociales. In J.L. Beauvois., R.V. Joule., \& J.M. Monteil (Eds). Perspectives cognitives et conduites sociales (pp. 143-150). Fribourg : Del Val.

HEIDER, F. (1958). The psychology of interpersonal relations. New York : Weiley.

JODELET, D. (1984). Représentations sociales : phénomènes, concept et théorie. In S. Moscovici. Psychologie sociale (pp. 357-378). Paris : PUF.

KIESLER, C.A. (1971). The psychology of commitment. New York : NY Academic Press.

LAHIRE, B. (1998). L'homme pluriel. Paris : Nathan.

LAutier, N. (2001). Psychosociologie de l'éducation. Paris : Armand Colin.

MiAs, C. (1998). L'implication professionnelle dans le travail social. Paris : L'Harmattan.

MoscovicI, S. (1961). La psychanalyse, son image et son public. Paris : PUF.

PAQUAY, L., \& WAGNER, M.C. (1996). Compétences professionnelles privilégiées dans les stages et en vidéo-formation. In L. Paquay., M. Altet., E. Charlier \& P. Perrenoud. (Eds.) Former des enseignants professionnels (pp. 154-179). Bruxelles : De Boeck.

PeYronie, H. (1998). Instituteurs : des maîtres aux professeurs d'école. Paris : PUF.

Roux-PEREZ, T. (2001). Des processus de construction de l'identité professionnelle des enseignants d'EPS : entre appartenance au groupe, expériences professionnelles singulières et recompositions identitaires à l'échelle du temps. Thèse de doctorat non publiée, Université de Nantes. 
ROUX-PEREZ, T. (2003). Identité professionnelle et modes d'implication privilégiés chez les enseignants d'EPS. Revue Les Sciences de l'Education pour l'Ere Nouvelle, $36,4,37-68$.

RouX-PEREZ, T. (2004). L'identité professionnelle des enseignants d'EPS: entre valeurs partagées et interprétations singulières. Revue STAPS, 63, 75-88.

Roux-Perez, T. (in press). Processus de construction de l'identité professionnelle des enseignants d'Education Physique et Sportive. Recherche et Formation. 43.

TAP, P. (1980). Identité individuelle et personnalisation, identité collective et changements sociaux. Toulouse : Privat.

TAP, P. (1998). Marquer sa différence. In J.C. Ruano-Borbalan. L'identité : l'individu, le groupe, la société (pp. 65-68). Auxerre : Sciences Humaines. 